\title{
The Components of Cacalia tangutica
}

\author{
Zhen-Ling Liı and Xuan Tian* \\ College of Chemistry and Chemical Engineering, State Key Laboratory of Applied Organic Chemistiy. \\ Lanzhou University, Lanzhou 730000. China \\ Received January 12, 2004
}

Key Words : Sesquiterpene, Eremophilane, Cacalia tanguhica, Antitumor activity

The genus Cacalia belongs to the tribe Senecioneae with more than 60 species occurring in China. ' of which $\mathrm{co} 26$ have long been used as Chinese traditional folk herbs. ${ }^{2}$ Several species of genus Cacalia have been investigated due to their antioxidant, antiradical and anti-histamine activities." The presence of pyrrolizidine alkaloids and sesquiterpenes in many species of the tribe Senecioneae is well documented. ${ }^{4-9}$ Recently, we have investigated the chemical constituents of Cacalia tangutica (Senecioneae). From a methanol extract of the root, one new eremophilane sesquiterpene, 12-hydroxy-3 $\alpha$-angeloyloxy-eremophila-6,9dien-8-one (1) combined with eight known compounds: one sesquiterpene, petasine (2), two triterpenes, $\alpha$-amyrin (3) and ursolic acid (4), one isopentenyl acetophnon derivative, hydroxytremetone (5), three cumarins, daphnetol (7,8-

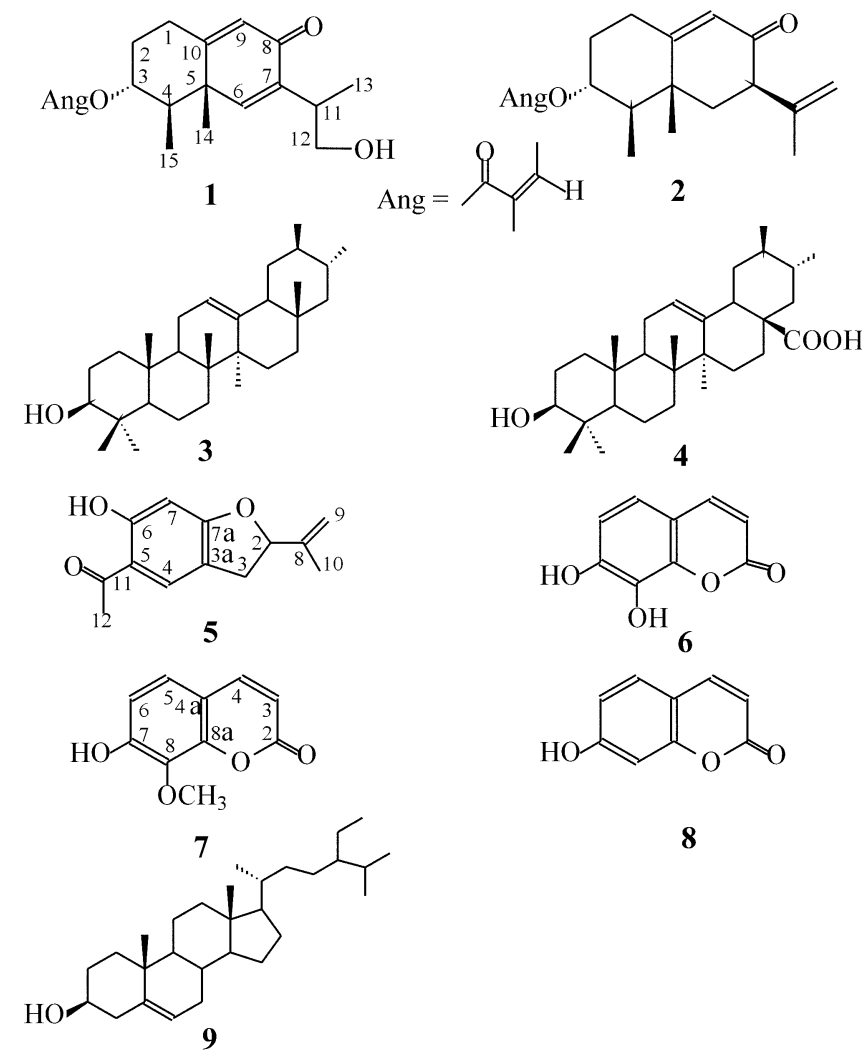

Compounds 1-9

"Corresponding Author. Fax: +86-93 l-89|2582, e-mail: xuant (a)lzu.edu.cn dihydroxycumarin) (6), hydrangetin (7), umbelliferone (8), one steroid. $\beta$-sitosterol (9) were isolated. The structure of the neo-sesquiterpene was elucidated by means of ${ }^{\prime} \mathrm{H}$ and ${ }^{1.3} \mathrm{C}$ NMR spectroscopic studies, including long rang correlation spectra with inverse detection (HMBC), ${ }^{\prime} \mathrm{H}-{ }^{\prime} \mathrm{H}$ COSY, NOE and NOESY.

Compound 1, a yellow oil. $[\alpha]_{\Gamma}^{21)}-26^{\circ}\left(c^{2} \quad 1.03, \mathrm{CHCl}_{3}\right)$, has the molecular formula $\mathrm{C}_{20} \mathrm{H}_{28} \mathrm{O}_{4}$ (HR-ESIMS: $m / z$ $333.2064[\mathrm{M}-\mathrm{l}]$ ', calcd. for $\left.\mathrm{C}_{20} \mathrm{H}_{28} \mathrm{O}_{4} 333.2060\right)$. Its $\mathrm{IR}$ and UV spectra show the presence of $\alpha \beta$-unsaturated carbonyl system-a ketone $\left(1663 \mathrm{~cm}^{-1}\right.$ and $\left.\lambda_{\text {па: }} 243 \mathrm{~nm}\right)$ and an ester (1715 and $1233 \mathrm{~cm}^{-1}$ ). The spectral data also indicated that the fourth oxygen atom seemed to be an alcohol $(3395$ $\mathrm{cm}^{-1}$ ). The existence of a ( $L$ )-2-methyl-2-butenoate (angeloyloate) moiety as well as the ester group in 1 , was inferred from the NMR signals, $\left[\delta_{\mathrm{H}} 6.10 \mathrm{~m}(\mathrm{lH}) .1 .95 \mathrm{dq}(J=7.6 \mathrm{~Hz}\right.$. $1.4 \mathrm{~Hz}, 3 \mathrm{H}), 1.86$ br s $(3 \mathrm{H}) ; \delta \cdot 167.5 \mathrm{~s}, 127.7 \mathrm{~s}, 138.5 \mathrm{~d}, 20.5$ q. $] 4.2$ q], by analogy with those of the constituents. ${ }^{10.11}$

The ${ }^{1} \mathrm{H}$ and ${ }^{13} \mathrm{C}$ NMR spectra of 1 were similar to those of 8-one-eremophila-6,9-diene derivatives reported in the literature except the primary alcohol group and secondary angeloyloate ester. ${ }^{1(1-1+1}$ A comparison of the 'H NMR spectral data with those of the corresponding $1 \beta$-hydroxy derivative $^{12}$ indicated that the $\mathrm{C}-\mathrm{I}$ position was not substituted. The downfield shifted signal of $\mathrm{H}-3$ was due to the 3-angeloyloxy while the signal $\delta 5.01 \mathrm{td}(J=11.2 \mathrm{~Hz} .4 .8$ $\mathrm{Hz}, \mathrm{H}-3$ ) showed that the angeloyloxy was $\alpha$-oriented. ${ }^{14-18}$ These were confirmed by the correlations between $\mathrm{H}-2$ and $\mathrm{H}-4$ with $\mathrm{H}-3$ in the ${ }^{\prime} \mathrm{H}-{ }^{\prime} \mathrm{H}$ COSY spectrum and the correlation between $\mathrm{H}-3$ and $\mathrm{H}-\mathrm{l} 4$ in the NOESY spectrum respectively. In the NOE spectrum of 1 , the NOE's [H- 15 with $\mathrm{H}-14(31.0 \%)$ and $\mathrm{H}-3(1.8 \%)]$ and $[\mathrm{H}-3$ with $\mathrm{H}-14$ $(3.0 \%)$ and $\mathrm{H}-15(1.5 \%)]$ were also appeared. According to the methylene signal $\delta 60.4$ in ${ }^{1.3} \mathrm{C}$ NMR spectra (DEP'l), the signal at $\delta 4.13 \mathrm{dd}(J=14.3 \mathrm{~Hz}, 6.8 \mathrm{~Hz}, \mathrm{H}-12)$ in ${ }^{\prime} \mathrm{H}$ NMR was assigned to the two protons attached to the carbon atom ( $\delta$ 60.4) bearing primary alcohol group. This was then supported by the cross placed between $\mathrm{H}-\mathrm{Il}$ and $\mathrm{H}-\mathrm{I} 2$ in the ${ }^{\mathrm{H}} \mathrm{H}-{ }^{\mathrm{j}} \mathrm{H} \mathrm{COSY}$.

In the HMBC spectrum, $\mathrm{H}-9(\delta 6.10)$ was correlated with $C-1(\delta 30.1), \mathrm{C}-2(\delta 29.7), \mathrm{C}-5(\delta 43.0)$ and $\mathrm{C}-7(\delta 141.4)$. $\mathrm{H}-6(\delta 6.92)$ was correlated with $\mathrm{C}-4(\delta 44.5), \mathrm{C}-5(\delta 43.0)$, C-7 $(\delta 141.4), C-8(\delta 187.7), C-10(\delta 166.1)$ and $\mathrm{C}-14(\delta$ 18.5). H-14 ( $\delta 1.23)$ was correlated with $\mathrm{C}-4(\delta 44.5), \mathrm{C}-5(\delta$ $43.0), \mathrm{C}-6(\delta 147.8)$ and $\mathrm{C}-10(\delta 166.1) . \mathrm{H}-15(\delta 1.14)$ was correlated with $\mathrm{C}-3(\delta 72.6), \mathrm{C}-4(\delta 44.5)$, and $\mathrm{C}-5(\delta 43.0)$, 
$\mathrm{H}-13(\delta 1.26)$ was correlated with $\mathrm{C}-12(\delta 60.4)$ and $\mathrm{C}-7(\delta$ $1+1.4), \mathrm{H}-3(\delta 5.01)$ was correlated with the carboxylic carbon of angeloyloxy $(\delta 167.5)$. It was determined that compound 1 was 12 -hydroxy-3 $\alpha$-angeloyloxy-eremophila6,9-dien-8-one.

Eight known compounds 2-9 were the results after repeated columun chromatography of the methanol extract of the root of Cacalia tangutica and were deduced by spectral data as petasine (2). ${ }^{13}$ two triterpenes, $\alpha$-amyrin $(3)^{3 \hat{i}}$ and ursolic acid (4). ${ }^{3 i}$ one isopentenyl acetophnon derivative. hydrosytremetone $(5),{ }^{\hat{1}}$ three cumarins. daphuetol $(6), 3$ hydrangetin $(7), 3$ umbelliferone $(8) .{ }^{23}$ one steroid, $\beta$ sitosterol (9).

The compound 1 was tested for in vitro antitumor activity against BEL 7402 (human liver carcinoma) by the method of the cells stained with sulforhodamine B (SRB). ${ }^{2-4}$ Test plates were incubated for 3 days. $\mathrm{IC}_{\text {sij }}$ values were determined for compounds $1(20.70 \mu \mathrm{g} / \mathrm{mL})$. This result showed that compound 1 was able to inhibit the growth of BEL 7402 with $\mathrm{IC}_{5 i j}$ values below $100 \mu \mathrm{g} / \mathrm{mL}$

\section{Experimental Section}

General Methods. IR spectra were measured on a Nicolet AVATAR 360 FT-IR instrument $\mathrm{KBr}$. UV spectra was measured on a Shimadzu UV-260 spectrometer. ID and 2D NMR spectrometer were measured on a Bruker AM-400FTNMR spectrometer and a Varian Mercury-300BB NMR spectrometer with TMS as inernal standard. HRESI-MS were recorded on a Bruker APEX II, EI-MS on a HP 5988A $\mathrm{GC} / \mathrm{MS}$ instrument. Optical rotations were measured using Perkin Elmer Model 34l. Silica gel (200-300 mesh) was used for $\mathrm{CC}$, silica $\mathrm{GF}_{3 \leq+}(10-40 \mu)$ for TLC were supplied by the Qingdao Marino Chemical factory. Qingdao, P. R. China. Spots were detected on TLC under UV lamp or by heating after spraying with $5 \% \mathrm{H}_{5} \mathrm{SO}_{4}$ in $\mathrm{C}_{3} \mathrm{H}_{3} \mathrm{OH}(\mathrm{v} / \mathrm{v})$.

Plant Material. The root of Cactia tangutica. were

Table 1. The NMR data of $1\left(400 \mathrm{MHz}, \mathrm{CDCl}_{3}\right)$

\begin{tabular}{rcll}
\hline No. & $\delta_{-}(\mathrm{DEPT})$ & \multicolumn{1}{c}{$\delta_{\mathrm{H}}$} & HMBC (carbon $)^{\alpha .}$ \\
\hline 1 & $30.1\left(\mathrm{CH}_{2}\right)$ & $2.33 \mathrm{~m}, 2.60 \mathrm{~m}$ & $(2), 3,8,9,(10)$ \\
2 & $29.7\left(\mathrm{CH}_{2}\right)$ & $1.60 \mathrm{~m}, 2.20 \mathrm{~m}$ & $(3), 4,9,10$ \\
3 & $72.6(\mathrm{CH})$ & $5.01 \mathrm{dd}(11.2,4.8)$ & $(4), \mathrm{OAng}(167.5)$ \\
4 & $44.5(\mathrm{CH})$ & $1.69 \mathrm{~m}$ & $(3),(5), 14$ \\
5 & $43.0(\mathrm{C})$ & & \\
6 & $147.8(\mathrm{CH})$ & $6.92 \mathrm{~s}$ & $4,(5),(7), 8,10), 14$ \\
7 & $141.4(\mathrm{C})$ & & \\
8 & $187.7(\mathrm{C})$ & & $1,2,5,7$ \\
9 & $125.3(\mathrm{CH})$ & $6.10 \mathrm{~m}$ & \\
10 & $166.1(\mathrm{C})$ & & $(12)$ \\
11 & $21.0(\mathrm{CH})$ & $1.26 \mathrm{~m}$ & 7,13 \\
12 & $60.4\left(\mathrm{CH}_{2}\right)$ & $4.13 \mathrm{dd}(14.3,6.8)$ & 7,12 \\
13 & $28.9\left(\mathrm{CH}_{3}\right)$ & $1.26 \mathrm{~m}$ & $4,(5), 6,10$ \\
14 & $18.5\left(\mathrm{CH}_{3}\right)$ & $1.23 \mathrm{~s}$ & $3,(4), 5$ \\
15 & $15.8\left(\mathrm{CH}_{3}\right)$ & $1.14 \mathrm{~d}(6.8)$ & \\
\hline
\end{tabular}

${ }^{\circ}$ Two-bond correlations are indicated in parentheses. collected in Minhe county, Qinhai province of China in October 1997. and identified by Prof. JiZhou Sun of Department of Biology. Lanzhou University. A voucher specimen (NO. 0108298) is deposited in Department of Biology. Lanzhou Univerisity.

Extraction and Isolation. Dried, powdered root $(1000 \mathrm{~g})$ of Cacalia tangutica were extracted with methanol by percolation at room temperature to give a residue $(102 \mathrm{~g})$ after evaporation. This residue was separated on $\mathrm{CC}$ over $1000 \mathrm{~g}$ silica gel with a gradient of petroleum ether $\left(60-90^{\circ}\right)$ ethyl acetate as eluent. Compound 1 was isolated during elution with petroleum ether $\left(60-90^{\circ}\right)$-ethyl acetate $(8: 1)$. Prep. tlc of eluates $5-7$ with $\mathrm{C}_{6} \mathrm{H}_{6}-\mathrm{E}$ mig of 1 .

Compound 1: $\mathrm{C}_{210} \mathrm{H}_{28} \mathrm{O}_{4}$. yellow oil; $[\alpha]_{D}^{20}:-26^{\circ}\left(\mathrm{CHCl}_{3}\right.$, c 1.03): IR $v: 3395,2923,1715,1663,1617,1457.1265$. $1233 \mathrm{~cm}^{-1}$ : UV $\lambda_{\text {thax }}^{\text {MeGi }}: 243 \mathrm{~mm}$ : HR-ESIMS: $m z 333.2064$ $[\mathrm{M}+\mathrm{l}]^{-}$, calcd. 333.2060 for $\left[\mathrm{C}_{20} \mathrm{H}_{28} \mathrm{O}_{4}+\mathrm{H}\right]^{-}$: EI-MS: $m z(\%$ $\div$ 10) $332.05[\mathrm{M}]^{+}$(3), $317\left[\mathrm{M}-\mathrm{CH}_{3}\right]^{-}$(203), 249 [M-Ang] $^{-}$ (3), 217 [317- HOAng] $^{-}$(199), 83 [Ang] $^{-}$(938). 43 (1000); ${ }^{1} \mathrm{H}$ and ${ }^{13} \mathrm{C}$ NMR (CDCl, $400 \mathrm{MHz}$ ) see Table 1

Compound 2: $\mathrm{C}_{20} \mathrm{H}_{38} \mathrm{O}_{3}$, colorless oil: EI-MS: $m z(\%)$ $316.20[\mathrm{M}]^{-}$(3), $233[\mathrm{M} \cdot \mathrm{Ang}]^{-}$(1), $216\left[233-\mathrm{H}_{2} \mathrm{O}\right]^{+}$(ll). 201 [216- $\left.\mathrm{CH}_{3}\right]^{-}(10) .148(100), 83$ [Ang] $^{-}(13){ }^{-1} \mathrm{H}$ NMR ( $\mathrm{CDCl}_{3} .400 \mathrm{MHz}$ ): $\delta 2.40$ (lH. m. H-l), 2.53 (lH. m. H-1). $1.50(\mathrm{lH}, \mathrm{m} . \mathrm{H}-2)$. $2.00(\mathrm{lH} . \mathrm{m}, \mathrm{H}-2) .4 .92$ (lH. ddd,$J=11$. $11,4.5 \mathrm{~Hz} . \mathrm{H}-3), 2.02(\mathrm{lH}, \mathrm{m} . \mathrm{H}-4) .2 .00(\mathrm{lH} . \mathrm{m}, \mathrm{H}-6) .2 .30$ (lH. m, H-6). $3.12(1 \mathrm{H}, \mathrm{dd}, J=14,5.0 \mathrm{~Hz} . \mathrm{H}-7), 5.79(1 \mathrm{H}$, d. $J=1.6 \mathrm{~Hz} . \mathrm{H}-9), 4.82$ (lH, s. H-12). 4.99 (lH. dq. $J=1.2$ Hz. H-12). 1.74 (3H. s, H-13). 1.24 (3H, s. H-14), $0.97(3 \mathrm{H}$. d. $J=6.8 \mathrm{~Hz}, \mathrm{H}-15)$. OAng: $\delta 6.10(\mathrm{lH}$. qq. $J=7.2 .1 .4 \mathrm{~Hz})$. $1.89(3 \mathrm{H}, \mathrm{m}) .1 .96(3 \mathrm{H}, \mathrm{m}) ;{ }^{12} \mathrm{C}$ NMR $\left(\mathrm{CDCl}_{3,} 400 \mathrm{MHz}\right): \delta$ 31.6 (C-1), 30.6 (C-2), 73.0 (C-3), 47.3 (C-4), 40.0 (C-5), 41.7 (C-6), 50.3 (C-7), $198.4(\mathrm{C}-8) .124 .6(\mathrm{C}-9), 167.6(\mathrm{C}-$ 10). $143.3(\mathrm{C}-11) .114 .4$ (C-12). $20.6(\mathrm{C}-13), 10.52(\mathrm{C}-14)$, 15.7 (C-15). OAng: $\delta 166.7(\mathrm{C}) .127 .9(\mathrm{C}), 138.0(\mathrm{CH}) .17 .2$ $\left(\mathrm{CH}_{3}\right) .20 .0\left(\mathrm{CH}_{3}\right)$.

Compound 3: $\mathrm{C}_{3 i j} \mathrm{H}_{50} \mathrm{O}$, white needle $(\mathrm{MeOH})$. m.p. 184$186^{\circ} \mathrm{C}$ : EI-MS: $m z(\% \div 100) 426.45[\mathrm{M}]^{+}(533), 411[\mathrm{M}-$ $\left.\mathrm{CH}_{3}\right]^{-}$(188). 218 (10000). 203 (3958), 189 (2912): ${ }^{13} \mathrm{C}$ NMR (DEPT. $\left.\mathrm{CDCl}_{3}, 400 \mathrm{MHz}\right): \delta 38.8$ (C-1). 27.3 (C-2). 79.1 (C-3). $38.8(\mathrm{C}-4) .55 .2(\mathrm{C}-5) .18 .4(\mathrm{C}-6) .33 .0(\mathrm{C}-7) .40 .0$ (C-8). 47.7 (C-9). 36.9 (C-10). 23.4 (C-11). 124.4 (C-12). 139.6 (C-13). 42.1 (C-14). 28.7 (C-15). 26.6 (C-16). 33.8 (C-17). 59.1 (C-18). 39.7 (C-19). 39.6 (C-20). 31.3 (C-21). 41.5 (C-22). 28.1 (C-23). 15.6 (C-24). 15.7 (C-25). 16.9 (C26). 23.3 (C-27). 28.1 (C-28). 17.5 (C-29). 21.4 (C-30).

Compound 4: $\mathrm{C}_{3 i 1} \mathrm{H}_{48} \mathrm{O}_{3}$. white powder $(\mathrm{MeOH})$. m.p 262-264 ${ }^{\circ} \mathrm{C}$ : EI-MS: $m z(\% \div 10) 456.30[\mathrm{M}]^{-}(26), 438$ (320), 423 (461). $410(57), 300(18), 248(1000) .203(500)$. 189 (104). $133(261)$.

Compound 5: $\mathrm{C}_{13} \mathrm{H}_{14} \mathrm{O}_{2}$, colorless oil: EI-MS: $m z(\% \div$ 100) 217.95 [M] $]^{-}$(3766). $203\left[\mathrm{M}^{\left.-\mathrm{CH}_{3}\right]^{-}}\right.$(3682). 175 (2579). 119 (6859). 117 (6747), 43 (10000): ' $\mathrm{H}$ NMR ( $\mathrm{CDCl}_{3 .} 400$ MHz): $\delta 5.27$ (1H. t. $J=8.0 \mathrm{~Hz}, \mathrm{H}-2) .2 .95(1 \mathrm{H}, \mathrm{dd} . J=9.6$. $15.0 \mathrm{~Hz} . \mathrm{H}-3) .3 .31(1 \mathrm{H}$. dd. $J=8.4 .15 .0 \mathrm{~Hz} . \mathrm{H}-3) .7 .50$ (1H. s. H-4). 6.38 (1H. s. H-7). 4.95 (1H. s. H-9). $5.09(1 \mathrm{H}$. 
s. $\mathrm{H}-9), 1.76(3 \mathrm{H}, \mathrm{s}, \mathrm{H}-10), 2.55(3 \mathrm{H}, \mathrm{s}, \mathrm{H}-12), \mathrm{OH}: 13.0$ $(1 \mathrm{H}, \mathrm{s}):{ }^{13} \mathrm{C} \mathrm{NMR}\left(\mathrm{CDCl}_{3}, 400 \mathrm{MHz}\right): \delta 87.6(\mathrm{C} \cdot 2), 33.2(\mathrm{C}-$ 3), 113.8 (C-3a), 126.6 (C-4), 143.2 (C-5), 165.8 (C-6), 98.1 (C-7). 166.6 (C-7a). $118.6(\mathrm{C}-8), 112.7$ (C-9). $17.0(\mathrm{C}-10)$. $201.9(\mathrm{C}-11) .26 .2(\mathrm{C}-12)$

Compound 6: $\mathrm{C}_{9} \mathrm{H}_{6} \mathrm{O}_{4}$. pale-yellow needle $(\mathrm{MeOH})$, m.p. $253.255^{\circ} \mathrm{C}$; EI-MS: $m z(\% \div 10) 177.90[\mathrm{M}]^{+}(1000), 150$ $\left[\mathrm{M}-\mathrm{H}_{2} \mathrm{O}\right]^{+}(770), 122(115) .69$ (329). $43(470):{ }^{1} \mathrm{H}$ NMR $\left(\mathrm{Me}_{2} \mathrm{CO}, 400 \mathrm{MHz}\right): \delta 6.13(\mathrm{lH}, \mathrm{d}, J=9.5 \mathrm{~Hz}, \mathrm{H} \cdot 3) .7 .83$ $(1 \mathrm{H}, \mathrm{d}, J=9.5 \mathrm{~Hz} . \mathrm{H}-4), 7.0 \mathrm{l}(1 \mathrm{H}, \mathrm{d}, J=8.0 \mathrm{~Hz}, \mathrm{H}-5), 6.85$ $(1 \mathrm{H}, \mathrm{d}, J=8.0 \mathrm{~Hz}, \mathrm{H}-6):{ }^{13} \mathrm{C}$ NMR $\left(\mathrm{Me}_{2} \mathrm{CO} .400 \mathrm{MHz}\right): \delta$ 161.3 (C-2). 113.1 (C-3), 145.6 (C-4). 113.4 (C-4a). 119.8 (C-5). 112.4 (C-6). 150.1 (C-7). 132.8 (C-8). 144.4 (C-8a).

Compound 7: $\mathrm{C}_{10} \mathrm{H}_{8} \mathrm{O}_{4}$, colorless needle $(\mathrm{MeOH})$. m.p. 154-156 ${ }^{\circ} \mathrm{C}$; EI-MS: $m z(\%) 191.95[\mathrm{M}]^{+}(1000), 177$ [M$\left.\mathrm{CH}_{3}\right]^{-}(770), 164(21), \mathrm{l} 49(30), 121(22), 93(13), 65(49)$. $39(20) ;{ }^{1} \mathrm{H}$ NMR $\left(\mathrm{CDCl}_{\mathrm{s} .}+400 \mathrm{MHz}\right): \delta 6.26(\mathrm{lH}$. d. $J=9.4$ Hz, H-3). 7.65 (lH. d. $J=9.4 \mathrm{~Hz}, \mathrm{H}-4$ ). 7.13 (lH. d. $J=8.5$ Hz, H-5). $6.9 \mathrm{l}(1 \mathrm{H}, \mathrm{d}, J=8.5 \mathrm{~Hz} . \mathrm{H}-6), 4.12(3 \mathrm{H}$. s. H$\left.\mathrm{OCH}_{3}\right) ;{ }^{13} \mathrm{C} \mathrm{NMR}\left(\mathrm{CDCl}_{3,}, 400 \mathrm{MHz}\right): \delta 160.4(\mathrm{C}-2), 112.6$ (C-3). 144.3 (C-4), 113.2 (C-4a), 123.2 (C-5). 112.2 (C-6). 152. I (C-7). 133.7 (C-8), 147.2 (C-8a); OCH $\mathrm{H}_{3}$ : 61.7 .

Compound 8: $\mathrm{C}_{3} \mathrm{H}_{6} \mathrm{O}_{3}$, pale-yollow needle $\left(\mathrm{Me}_{2} \mathrm{CO}\right)$. m.p. $230-232{ }^{\circ} \mathrm{C}$; EI-MS: $m z(\% \div 10) 161.95[\mathrm{M}]^{+}(1000)$. 134 (997), 105 (332). 78 (351); ${ }^{1} \mathrm{H}$ NMR (Me, CO. 400 MHz): $\delta 6.14$ (lH. d, $J=9.2 \mathrm{~Hz}, \mathrm{H}-3$ ). 7.85 (lH. d. $J=9.2$ $\mathrm{Hz}, \mathrm{H}-4), 7.48(1 \mathrm{H}, \mathrm{d} . J=8.4 \mathrm{~Hz}, \mathrm{H}-5), 6.82(\mathrm{lH}, \mathrm{dd} . J=$ 8.4. $2.3 \mathrm{~Hz}, \mathrm{H}-6), 6.73(1 \mathrm{H}, \mathrm{d}, J=2.3 \mathrm{~Hz} . \mathrm{H}-8) ;{ }^{13} \mathrm{C}$ NMR $(\mathrm{Me}, \mathrm{CO}, 400 \mathrm{MHz}): \delta 161.4(\mathrm{C}-2), 112.6(\mathrm{C}-3) .144 .8(\mathrm{C}-$ 4), 112.6 (C-4a). 130.4 (C-5), 113.8 (C-6), 162.2 (C-7). 103. 1 (C-8). $156.8(\mathrm{C}-8 \mathrm{a})$.

Compound 9: $\mathrm{C}_{39} \mathrm{H}_{5 j} \mathrm{O}$. colorless needle $\left(\mathrm{Me}_{2} \mathrm{CO}\right)$, m.p. 138-140 ${ }^{\circ} \mathrm{C}$; EI-MS: $m z(\%) 414.30[\mathrm{M}]^{+}(26), 396(10), 381$ (5). $329(9) .303(7), 273(6), 255$ (11), $213(10) .199(5), 159$ (11), $145(18) .95(28), 81(40) .43(100)$.

Acknowledgment. We would like to thank Prof. Ji-zhou Sun of Department of Biology. Lanzhou University for the authentication of the plant.

\section{References}

1. Institute of Botany Iconographia Comophytonm Sinicontm: Science Press: Beijing. 1973: p 795.

2. The China Medicinal Materials Co.; Zhonggno Zhongrao Zinzan Zhivao: Science Press: Beijing. 1994; 1271.

3. Krasovskava. N. P.: Kulesh, N. I.; Denisnko, V. A. Khim. Prit: Soedin 1989.643

4. Sugama. K.: Hayashi. K: Mitsuhashi. H. Phvtochemisov 1985. 24. 1531 .

5. Gonzales. U. J.: Bassabe B. P.; Sanchez. M. I.; Fenandez, M. R.: Lopez. E. M.: Fernandez, R. A. Phwochemistry 1988. 27. 1507.

6. Pieters. L. A.: Vlietnick. A. J. Planta 1ed. 1988. 54. 178

7. Khetwal. K. S.: Maural. K. Planta Hed. 1988. 54. 188.

8. Bolmant1. F.: Zdero. C.: Jakupovic. J.: Misra. L. N.: Benerjee. S.: Singh. P.: Bartah. R. N.; Metwally: M. A.; Schmeda-Hirschmann. G.; Vicent, L. P. D; King, R. M; Robinson, H. Phyochemistry 1985. 24, 1249

9. Aclinou. P.: Betnkouider. A.: Massiot. G.: Lemen-Olivier. L. Phutochemisny 1991. 30.2083

10. Zhang. S. M.: Zhao. G. L.: Li. R.: Lin. G. Q. Phwochemistry $1998 .+8,519$

11. Zhao. Y.: Peng, H.: Jia, Z. J. J. Nat. Products 1994. 57. 1626.

12. Zhao. Y.: Jia. Z. T.: Yang. L. Plana Hed. 1994. 60.91

13. Takagi. I.: Gakuin. Y.: Naya. K. Chem. Pham. Bull. 1977. 50. 3320 .

14. Dupre. S.: Grenz, M:- Jakupovic, J; Bohlmann, F; Niemeyer, H M. Plntochemistry 1991,30.1211

15. Bohlmann. F.: Suwita. A.: Zdero. C. Plytochemisny 1978. 17. 1763.

16. Zdero. C.: Bohlmant1. F.: Liddell. J. R. Phytochemistry 1989. 28. 3532 .

17. Bohlmann, F.: Ates, N:; King. R. M.; Robinson, H. Plntochentism 1983. 22, 1675.

18. Bohlmann. F: Ehlers. D.: Zdero. C. Phytochemisty 1978, 17. 467.

19. Bohlmnant1. F.: Zdero. C. Phytochemisty 1978. 17. 1337.

20. Mahato. S. B.: Kundu. A. P. Phtochemism 1994.37. 1517.

21. Bohlmann. F: Grenz, M. Chent. Ber. 1970, 103.90.

22. Gray, A. I.: Meegan. C. J:- O'Callaghan, N. B. Phwochemistry 1987. 26,257

23. Chatterjee. A.: Sarkar. S.: Shoolery. J. N. Phytochemisoy 1980. 19. 2219.

24. Lee. S. K.: Nam. K. A.: Heo. Y. H. Plonta Med. 2003. 69. 21. 